\title{
Vates Apollinis, vates Augusti - Das Verhältnis des palatinischen Apollonheiligtums zu Orakeln und sein Einfluss auf das Selbstverständnis der zeitgenössischen Dichter
}

Jens Fischer

(Martin-Luther-University Halle-Wittenberg)

\begin{abstract}
Vates Apollinis, vates Augusti - The Relationship of the Palatine Sanctuary of Apollo to Oracles and its Influence on the Self-image of Contemporary Poets
\end{abstract}

\begin{abstract}
The sanctuary of Apollo on the Palatine is one of the most important Augustan buildings. Situated very close to the first emperor's private residence, it is central for our understanding of Augustan culture. As one of its main functions, from at least 12 BCE the temple of Apollo accommodated the libri Sibyllini, one of Rome's most precious religious possessions. Another part of the sanctuary was a prestigious library that featured a Latin and a Greek section and played an important role in the lives of the contemporary poets. By means of an interdisciplinary approach, I demonstrate that the combined influence of these two functions of the Palatine sanctuary can still be recognized today in the fact that the Augustan poets started to call themselves vates. It will be shown that a combination of historical, archeological, and philological research can lead to a better understanding of this interesting phenomenon of the Augustan epoch which until now has almost only been approached from a philological point of view.
\end{abstract}

\section{Keywords}

Apollo Palatinus; Sibylline Oracles; vates; quindecimviri; Augustus; roman libraries; Augustan poetry; Virgil; Horace; Propertius; Tibullus; Ovid 


\section{Einleitung}

Bei dem palatinischen Tempel des Apollon, dessen Pracht sich einst unmittelbar neben dem privaten Wohnsitz des ersten römischen Kaisers Augustus erhob und den der Caesarerbe im Jahr 36 v. Chr. nach seiner Rückkehr nach Rom im Anschluss an die von ihm gewonnene Schlacht bei Naulochus gegen Sextus Pompeius gelobte, ${ }^{1}$ handelt es sich um ein Gebäude mit einem für die gesamte Epoche ikonischen Charakter. Nicht selten erkennt die Forschung in ihm geradezu so etwas wie ein Symbol des damaligen Zeitgeistes. $^{2}$ Etwas Ähnliches gilt schon für die Zeitgenossen. Es existieren nur äußerst wenige Bauten, welche von den kontemporären Schriftstellern vergleichbar oft erwähnt werden. ${ }^{3}$ Möglicherweise die berühmteste dieser literarischen Bezugnahmen findet sich im 8. Buch der Aeneis des Vergil. Dort beschreibt der Dichter, wie Augustus während seines dreifachen Triumphzuges des Jahres 29 v. Chr. vor dem Tempel des Apollon Platz genommen hätte und diesen mit der Beute seiner Siege schmücke. ${ }^{4}$ Nicht zuletzt im Licht dieser Worte stehen zumeist die häufig postulierten militärischen Konnotationen des Monuments im Zentrum der wissenschaftlichen Aufmerksamkeit. ${ }^{5}$ Für den vorliegenden Beitrag sollen diese jedoch keine Rolle spielen. In seinem Rahmen soll vielmehr ein Aspekt des Gebäudes hervorgehoben werden, der von der modernen Forschung allzu häufig nicht gebührend berücksichtigt wird, obwohl er insbesondere für jene Zeitgenossen überaus präsent war, die aktiv an der literarischen Kultur partizipierten. Die Rede ist davon, wie der Tempel des Apollon mit der Divination und der Dichtkunst zwei zentrale Zuständigkeitsgebiete des Gottes miteinander verknüpfte und wie sich diese Eigenschaft des Monuments unmittelbar auf das Selbstverständnis der augusteischen Dichter auswirkte. ${ }^{6}$

\section{Die Verbindung des Heiligtums zur Literatur}

Die enge Verbindung des Tempels zur zeitgenössischen Literatur ist offenkundig. Sie manifestiert sich überaus konkret in der bekannten augusteischen Bibliothek, die einen wichtigen Bestandteil des palatinischen Gesamtkomplexes darstellte. Die beiden Gebäude, welche sich noch heute im archäologischen Befund abzeichnen, stammen jedoch aus

1 D.C. 49,15,5; Suet. Aug. 29,3; Vell. 2,81,3. Zanker (1983: p. 21); Hekster \& Rich (2006: pp. 151-152).

2 Lefèvre (1989: p. 11); Zanker (2009: pp. 90-91).

3 „The Temple of Apollo with its associated structures comes up for mention in Augustan poetry more often than any other urban monument" (White 1993: p. 321). Siehe auch: Miller (2002: p. 130); Miller (2009: p. 185).

4 Ipse, sedens niveo candentis limine Phoebi, dona recognoscit populorum aptatque superbis postibus; incedunt victae longo ordine gentes. (Verg. Aen. 8,720-723). Wobei es sich hier um einen ebenso berühmten wie deutlichen Anachronismus handelt, da der Tempel erst im Jahr 28 v. Chr. eingeweiht wurde; siehe Miller (2009: p. $65)$.

5 Siehe bspw.: Zanker (1983); Kellum (1985); Lefèvre (1989); Zanker (2009).

6 Graf (2009: pp. 28-64). 
domitianischer Zeit. ${ }^{7}$ Bei der augusteischen Bibliothek handelte es sich um ein einzelnes Gebäude, dessen Bücherbestand sich allerdings bereits ebenfalls auf eine lateinische und eine griechische Sektion verteilte. ${ }^{8}$ In Anbetracht der Prominenz, welche der Komplex schon alleine durch seine räumliche Nähe zu Augustus' privater Wohnstätte besaß, überrascht es kaum, dass er von den zeitgenössischen Autoren beinahe ebenso oft erwähnt wird wie der Tempel des Apollon selbst. In seinen Episteln etwa nimmt Horaz gleich mehrfach auf die Bibliothek Bezug und empfiehlt dem Prinzeps, welche Art von Autoren er für die Aufnahme berücksichtigen sollte. ${ }^{9}$ In einer unten noch etwas ausführlicher zu behandelnden Passage imaginiert wiederum Ovid das Heiligtum als Ziel allen Sehnens eines von ihm aus der Verbannung nach Rom zurückgesandten Buches. ${ }^{10}$ Noch von dem jüngeren Plinius erfahren wir von öffentlichen Lesungen, welche häufig an diesem Ort stattgefunden haben müssen. ${ }^{11}$ Balensiefen beschreibt den Komplex daher korrekt als eine Art „Literaturheiligtum“, in dem Apollon auch als „Gott der Bücher“ verehrt wurde. ${ }^{12}$ Eben dieses Heiligtum aber befand sich unmittelbar im Herzen Roms und war vor allem von den Rängen des Circus Maximus aus deutlich zu erkennen, welche in augusteischer Zeit bereits 150.000 Menschen Platz boten. ${ }^{13}$ Es ist daher sicherlich keine gewagte Hypothese, dass die mit ihm verbundenen Gegebenheiten einen festen Bestandteil des kontemporären stadtrömischen Diskurses bildeten.

\section{Die Verbindung des Heiligtums zur Divination}

Die unmittelbare Verbindung des Tempels zur Divination ist für den modernen Betrachter nicht mehr ganz so deutlich nachzuvollziehen. Doch lesen wir bei Sueton:

Postquam vero pontificatum maximum, quem numquam vivo Lepido auferre sustinuerat, mortuo demum suscepit, quidquid fatidicorum librorum Graeci Latinique generis nullis vel parum idoneis auctoribus vulgo ferebatur, supra duo milia contracta undique cremavit ac solos retinuit Sibyllinos, hos quoque dilectu habito; condiditque duobus forulis auratis sub Palatini Apollinis basi.

„Nachdem er aber das Amt des Pontifex Maximus, das er dem Lepidus, solange dieser lebte, keinesfalls hatte abnehmen wollen, nach dessen Tod schließlich übernommen hatte, ließ er alles, was an griechischen und lateinischen Weissagungsbüchern, deren Verfasser nicht bekannt

7 Suet. Aug. 29. Balensiefen (2002: pp. 102-105); Balensiefen (2004: pp. 103-111); Iacopi \& Tedone (2005/6: pp. 351-378); Tucci (2013: pp. 286-291); Fleury \& Madeleine (2019: pp. 171-179).

8 D.C. 53, 1; Suet. Aug. 29; vgl. auch: Front. ep. 4,5; Plin. nat. 7,210 und 34, 43; Suet. Gramm. 20. Dix (1988: p. 27); Davis (2001: p. 113); Balensiefen (2002: p. 110); Balensiefen (2009: p. 72); Hall (2014: p. 172).

9 scripta, Palatinus quaecumque recepit Apollo (Hor. epist. 1, 3, 17); verum age et his, qui se lectori credere malunt quam spectatoris fastidia ferre superbi, curam redde breuem, si munus Apolline dignum uis complere libris et uatibus addere calcar (Hor. epist. 2,1,214-217); spectemus uacuam Romanis uatibus aedem (Hor. Epist. 2,2,94). Babcock (1967: p. 191); Miller (2004: p. 180, Anm. 39); Nicholls (2018: p. 244).

10 Ov. trist. 3,1,59-68; siehe auch unten.

11 Plin. epist. 1,13; vgl. auch: Calp. ecl. 4,157-159; Hor. sat. 1,10,38; Schol. Hor. sat. 1,10,38.

12 Balensiefen (2002: p. 99); Balensiefen (2009: p. 72).

13 Humphrey (1986: p. 126); vgl. auch Künzl (1988: pp. 16-17). 
waren oder als zu wenig geeignet galten, in der Bevölkerung umlief, mehr als zweitausend Bände, von überall zusammentragen und verbrennen. Alleine die Sibyllinischen behielt er zurück, aber auch diese nur in Auswahl. Er bewahrte sie in zwei vergoldeten Schränken unter dem Sockel des Palatinischen Apollon auf.“

Suet. Aug. 31,1

Diese Worte behandeln das Jahr 12 v. Chr. Lepidus, der frühere dritte Mann im zweiten Triumvirat und amtierende Pontifex Maximus, war gerade verstorben. Augustus zögerte nicht lange, bevor er sich selbst in dieses höchste Priesteramt wählen ließ. Im Anschluss daran bestand laut Sueton eine seiner ersten Handlungen darin, alles, was an Orakelschriften (fatidicorum librorum) unter den Händen der Bevölkerung zirkulierte (vulgo ferebatur), einzuziehen und zu verbrennen. Er behielt nur jene zurück, denen eine sibyllinische Verfasserschaft zugeschrieben wurde, und verwahrte sie in zwei vergoldeten foruli unterhalb der Basis des Kultbildes des palatinischen Apollontempels. ${ }^{14}$ In diesen Handlungen kommt deutlich zum Ausdruck, dass der Tempel nicht nur in einem engen Bezug zur Divination stand, sondern zu einer sehr speziellen Form derselben - der sibyllinischen Orakelliteratur. ${ }^{15}$ Doch worum genau handelt es sich bei dieser?

Sibyllen zugeschriebene Orakeltexte waren eine Form von Literatur, die in der Antike durchaus eine gewisse Popularität besaß. Tatsächlich ist uns eine Kenntnis der Figur der Sibylle das erste Mal bereits für den Anfang des fünften vorchristlichen Jahrhunderts belegt. ${ }^{16}$ Von dieser Zeit an verbreiteten sich derartige Schriften praktisch über den gesamten mediterranen Raum. ${ }^{17}$ Das umfangreichste Zeugnis sibyllinischer Orakel, das der modernen Forschung erhalten geblieben ist, stellt die Sammlung der Oracula Sibyllina dar, welche jedoch gänzlich einer jüdisch-christlichen Traditionslinie angehört. ${ }^{18}$ Hinzu treten einige wenige Fragmente der paganen Sibyllendichtung. ${ }^{19}$ All diese Texte und Fragmente belegen, dass sibyllinische Orakel gleich welcher Tradition in Hexametern verfasst waren und somit als eine Form der Dichtung zu verstehen sind..$^{20}$

Auch Rom selbst besaß eine berühmte Sammlung derartiger Schriften. Der legendenhaften Überlieferung zufolge erwarb sie einer der letzten Könige unmittelbar von der Cumaeischen Sibylle. Die von mehreren Autoren wiedergegebene Erzählung beschreibt, wie die Prophetin zwei Drittel der von ihr nach Rom gebrachten Bücher verbrannte, bevor der König den Rest schließlich für denselben Preis erwarb, welchen er auch für

14 Miller (2009: p. 194).

15 Zudem wurden hier auch die Carmina Marciana aufbewahrt sowie Bücher, die der etruskischen Nymphe Begoe zugeschrieben wurden; siehe Serv. Aen. 6,72.

16 Plu. De Pyth. or. 6 = Heraclit. Frg. 22 B 92 DK. Parke (1988: pp. 63-64); Schröder (1990: pp. 145-146).

17 Graf (1985: pp. 339-340); Potter (1990: pp. 477-478).

18 Zu den Oracula Sibyllina siehe: Bremmer (1993: p. 167); Sfameni Gasparro (1998); Gauger (2002); Sfameni Gasparro (2002: pp. 61-112); Roessli (2004: pp. 60-65); Lightfoot (2007).

19 Gauger (2002: pp. 360-367); Lightfoot (2007: p. 3).

20 te duce Romanos numquam frustrata Sibylla, abdita quae senis fata canit pedibus. (Tib. 2,5,15 f.); vgl. auch bspw.: Paus. 7,8 und 10,12,3; Phleg. macr. 6,1; Plu. Dem. 19. 
alle Bücher hätte bezahlen können. ${ }^{21}$ Während der republikanischen Epoche wurden die sibyllinischen Orakel im Tempel des Jupiter auf dem Kapitol aufbewahrt. ${ }^{22}$ Augustus transferierte sie dann in den von ihm neu errichteten palatinischen Tempel. ${ }^{23}$ Verwaltet wurden die Bücher von den decem- bzw. quindecimviri sacris faciundis, einem der vier höchsten Priesterkollegien (quattuor amplissima collegia). ${ }^{24}$ Die Priester konsultierten die Orakelsprüche im Auftrag des Senats beim Auftreten der fürchterlichsten Vorzeichen. Sie entnahmen ihnen Ritualvorschriften, deren Ausführung die Götter wieder gewogen stimmen sollte. ${ }^{25}$ Diese Anweisungen bestanden nicht selten in der Einrichtung neuer Kulte, wodurch die Bücher die römische Religion und Kultur im Verlauf der republikanischen Epoche bleibend beeinflussten.

Dies bringt uns zurück zu Apollon, dem Gott des palatinischen Heiligtums. Abgesehen von der Tatsache, dass die sibyllinischen Bücher Roms seit augusteischer Zeit im Inneren dieses Tempels aufbewahrt wurden, existierte im zeitgenössischen Rom noch eine weitere wichtige Verbindung zwischen dem Gott und den Orakeln, welche von der Forschung allzu häufig nicht gebührend betont wird. Die Rede ist davon, dass sowohl in unseren literarischen als auch unseren numismatischen und archäologischen Quellen eine starke Assoziation der decem- bzw. quindecimviri mit Apollon zum Ausdruck kommt. Aufgrund der Bedeutung dieser Tatsache für die übergeordnete Argumentation des vorliegenden Beitrages sollen an dieser Stelle die wichtigsten Belege angeführt werden. ${ }^{26}$

Eine vollkommen unzweideutige Bezugnahme auf das enge Verhältnis der Priesterschaft zu dem Gott findet sich in der Biographie des jüngeren Cato des Plutarch. In dieser bezeichnet der Autor das Quindecimvirat expressis verbis als „Priesterschaft des

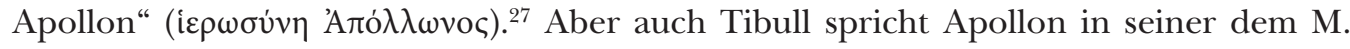
Valerius Messalla Messalinus gewidmeten und anlässlich von dessen Aufnahme in das Kollegium der quindecimviri verfassten Elegie 2,5 direkt an (Phoebe) und weist den Gott darauf hin, dass ein neuer Priester (novus sacerdos) seine Tempel (tua templa) betrete. ${ }^{28}$ Außerdem waren es die decem- bzw. quindecimviri, welche im Rahmen der jährlich

21 App. Reg. 9; D.H. 4,62; Gell. 1,19; Isid. Etym. 8,8; Lact. Inst. 1,6; Lyd. Mens. 4,47; Plin. nat. 13,88; Serv. Aen. 6,72; Solin. 2,16-18; Tz. ad Lyc. 1279; Zon. 7,11; Roessli (2004: p. 57).

22 D.H. 4,62,5; Lact. Inst. 1,6,14; Tac. ann. 6,12.

23 Suet. Aug. 31,1. Radke (1963: p. 1128); Balensiefen (2002: p. 98); Scheid (2009: p. 284); Miller (2009: p. 194); Wardle (2014: pp. 248-249).

24 Aug. civ. 6,3; Cic. har. resp. 18; Cic. leg. 2,20; Cic. nat. 3,5. Radke (1963: p. 1114); Février (2002: p. 821); Gillmeister (2019).

25 Gillmeister verwendet für den Vorgang den nicht unpassenden Begriff eines „state oracle“; Radke (1963: p. 118); Roessli (2004: pp. 57-60); Monaca (2005: pp. 31-35); Guittard (2007: pp. 243-245); Santi (2008); Keskiaho (2013: p. 156); Gillmeister (2015: p. 211); Gillmeister (2019: p. 126); Fischer (2020b).

26 Eine umfangreiche Analyse des engen Verhältnisses der Priesterschaft der quindecimviri zu dem Gott Apollon sowie eine Behandlung der wichtigen sich hieraus ergebenden Rückschlüsse besonders auf unser Verständnis der augusteischen Kultur stellen einen zentralen Aspekt der bisher noch unveröffentlichten Dissertation des Autors dieses Beitrages dar; Fischer (forthcoming); Siehe schon jetzt: Fischer (2020a) und Fischer (2020b: pp. 548-553).

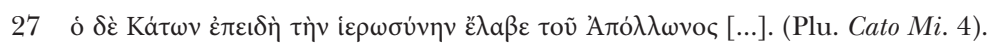

28 Phoebe, faue: nouus ingreditur tua templa sacerdos (Tib. 2,5,1). Miller (2009: pp. 234-235). 
stattfindenden ludi Apollinares das zentrale Opfer ausführten. ${ }^{29}$ Und auch Livius lässt Publius Decius Mus, den Konsul des Jahres 300 v. Chr., in einer fingierten Rede äußern, dass es sich bei der Priesterschaft um die antistites Apollinaris sacri handele. ${ }^{30}$ Dasselbe Bild kommt in der zeitgenössischen Münzprägung zum Ausdruck. Hinsichtlich dieser vertreten Numismatiker bereits seit langem die Ansicht, dass die Abbildung des apollinischen Dreifußes auf dem Revers eine Verbindung des zuständigen Münzmeisters mit dem Kollegium der decem- bzw. quindecimviri anzeige. ${ }^{31}$ Die sowohl schönste als auch wichtigste während des ersten vorchristlichen Jahrhunderts geprägte Münze ist dabei jene des Lucius Manlius Torquatus, welche auf ihrem Revers eben den Dreifuß zeigt, während sie auf ihrem Avers den Kopf der Sibylle abbildet. ${ }^{32}$ Doch auch zahlreiche andere Münzen werden auf dieselbe Weise interpretiert. ${ }^{33}$ Eine von ihnen, welche für das Verständnis des palatinischen Heiligtums besonders aussagekräftig ist, prägte Octavian im Jahr 37 v. Chr. Auch sie bildet auf ihrem Revers den Dreifuß ab. Auf ihrer Basis wird der Eintritt des späteren Augustus in das besagte Kollegium gewöhnlich in eben dieses Jahr datiert - das unmittelbare Vorjahr des Baubeschlusses des palatinischen Tempels. ${ }^{34}$ Zudem findet sich der Dreifuß aber auch in archäologischen Kontexten, welche eindeutig auf das Kollegium der quindecimviri hinweisen. So etwa ist er mittig zwischen simpulum und lituus, den Kennzeichen der Pontifices und der Auguren, auf einem heute in Köln aufbewahrten Glaskameo abgebildet. ${ }^{35}$ Ebenso sieht man ihn auf dem Weihrauchkästchen der Darstellung eines Opfergehilfen auf der Ara Pacis. ${ }^{36}$ Vor allem aber haben sich uns gleich mehrere marmorne Dreifußbasen erhalten, welche durch ihre Symbolik ebenfalls dem Priesterkollegium zugewiesen werden können. ${ }^{37}$ Die überaus starke Verbindung der quindecimviri zu Apollon wird vor dem Hintergrund zahlreicher Quellen somit evident. Für die zeitgenössischen Römer bildete sie demnach einen geradezu selbstverständlichen Teil ihres täglichen Lebens und Erlebens.

29 Liv. 25,12,9-10; Fischer (2020b: p. 561).

30 Liv. $10,8,2$.

31 Radke (1963: pp. 1139-1140); Siebert (1999: pp. 134-135); Ryan (2006/7: pp. 39-40).

32 RRC 411; Sydenham 835a.

33 RIC I Augustus 367 und 410, 4A; RIC I2 Vitellius 70 und 85 f. und 108 f.; RRC 254 und 498-500 und 502/2 und 511/1; Sydenham 475 und 1271-1274 und 1290 und 1346; Siebert (1999: pp. 130-136).

34 RRC 537/2. Gagé (1931: pp. 79-80); Simon (1986: p. 62); Beard \& North \& Price (1998: p. 186); Siebert (1999: pp. 134-135); Rüpke (2005: p. 839 mit Anm. 4); Hekster \& Rich (2006: p. 161); Balensiefen (2009: pp. 78-79); zu den Implikationen dieses starken chronologischen Zusammenhangs siehe ausführlich: Fischer (forthcoming); bereits jetzt: Fischer (2020a: pp. 133-134).

35 Köln, Römisch-Germanisches Museum Inv. 72,153; Simon (1986: p. 164 mit Abb. 214); Zwierlein-Diehl (2012: p. 128 mit Abb. 504 Tafel 109).

36 Simon (1986: p. 37 mit Abb. 42); Mlasowsky (2010: p. 52); Simon (2012: p. 31).

37 Paris, Louvre, Inv. MA 358; Rom, Musei Capitolini, Inv. 920; Rom, Museo Nazionale Romano - Terme di Diocleziano, Inv. 212207; Rom, Terme di Diocleziano, Inv. 669; Martini (1988: pp. 182-184); Götte (1984: pp. 574-585); Rendini (1988: pp. 233-236); Dräger (1994: pp. 212-213 und Kat. Nr. 40 mit Taf. 84-85); Zanker (2009: pp. 124-126 mit Abb. 99 a-c und 343). 


\section{Der Begriff des vates in der augusteischen Literatur}

Als Nächstes ist es notwendig zu betrachten, auf welche Art und Weise bereits Cicero in seiner im Jahre 56 v. Chr. gehaltenen Rede De haruspicum responso die Verbindung zwischen den sibyllinischen Büchern Roms und dem Gott Apollon hervorhebt. Er äußert dort:

Ego vero primum habeo auctores ac magistros religionum colendarum maiores nostros [...] qui [...] fatorum veteres praedictiones Apollinis vatum libris [...] contineri putaverunt.

„Als erstes aber erkenne ich Urheber und Lehrmeister der Kultangelegenheiten in unseren Vorvätern, [...] die glaubten, [...] die alten Vorhersagen des Schicksals seien in den Büchern der Seherinnen Apollons [...] enthalten.“

Cic. har. resp. 18

Anhand dieser Worte lässt sich feststellen, dass die von den decem- bzw. quindecimviri aufbewahrten sibyllinischen Bücher Roms von Cicero als vom Gott Apollon inspirierte Orakel verstanden wurden. Von noch größerem Interesse für die Argumentation des vorliegenden Beitrages aber ist, dass Cicero die Sibyllen als vates bezeichnet.

Ursprünglich bedeutet vates selbstverständlich nichts anderes als „Seher“ bzw. „Seherin“, weshalb es auf den ersten Blick kaum bemerkenswert erscheint, dass Cicero die Sibyllen eben als das bezeichnet, was sie dem allgemeinen Verständnis zufolge waren. ${ }^{38}$ Gleichzeitig ist vates aber auch ein Terminus, den antike Dichter des Öfteren zur Beschreibung ihrer selbst gebrauchten. Besonders charakteristisch ist die Verwendung des Begriffes dabei für die augusteische Epoche. Tatsächlich existieren nur überaus wenige Hinweise, dass das Wort bereits vor der frühen Kaiserzeit auf diese Weise genutzt worden wäre. Während der augusteischen Epoche wird es dann aber ein häufiges Synonym für poeta. ${ }^{39}$ Die Idee hinter der besagten Metapher ist die folgende: Wie der Seher so empfängt auch der Dichter seine Inspiration unmittelbar von einer Gottheit und zwar besonders von Apollon. ${ }^{40}$ Bereits in der älteren griechischen Dichtung findet sich des Öfteren eine ähnliche Verwendung der Worte $\mu a \dot{v \tau \iota \varsigma ~ o d e r ~ \pi \rho o \varphi n ́ \tau \eta ৎ . ~}{ }^{41}$ Vergil gebraucht vates in dieser Weise sowohl mehrmals in seinen Eklogen als auch in seiner Aeneis. ${ }^{42}$ Bei Horaz trifft man auf das Wort mit dem Bezug auf den Dichter in seinen Oden, Epoden und Episteln. ${ }^{43}$ Ein Ausspruch, welcher sich in der Ode 1,31 findet (Quid

38 Joceyln (1995: p. 20).

39 Tatsächlich ist eine Verwendung des Begriffs vates anstelle von poeta vor Varro (l.l. 7,3) in der lateinischen Literatur nicht belegt. Zu dem Phänomen siehe generell die folgenden Studien: Dahlmann (1948); Bickel (1951); Newman (1967); Della Casa (1995); Jocelyn (1995).

40 Della Casa (1995: pp. 60-61); Rüpke (2006: pp. 221-222); Le Doze (2016: p. 96).

41 Vgl. bspw.: Bakchyl. epin. 8,3; Pind. Fr. 94a 5-6 und Fr. 150; Pind. Pae. 6,6; Nisbet \& Hubbard (1970: p. 349); Nagy (1990: pp. 56-64).

42 Verg. Aen. 7,41; Verg. ecl. 7,25-28; Verg. ecl. 9,32-34.

43 Hor. carm. 1,31,1f. und 2,6,24 und 2,20,3 und 4,3,15 und 4,6,44 und 4,9,28; Hor. epist. 2,1,217 und 2,2,94; Hor. epod. 16,66 und 17,44. 
dedicatum poscit Apollinem vates?), ist dabei besonders hervorzuheben, da dieses Werk von der Forschung seit langem mit der Eröffnung des palatinischen Apollontempels in Verbindung gebracht wird. ${ }^{44}$ Aber auch Properz greift auf dieselbe Art und Weise auf das Wort zurück. ${ }^{45}$ Dasselbe trifft auf Ovid zu. ${ }^{46}$ Besonders aussagekräftig ist allerdings die oben bereits erwähnte Elegie 2,5 des Tibull. ${ }^{47}$ In diesem Gedicht kommt das Wort vates insgesamt genau dreimal vor. Zweimal (in Vers 18 und 65) bezieht es sich auf die Sibylle. In Vers 114 jedoch verweist es auf Tibull selbst. ${ }^{48}$ Spätestens anhand eben dieser Stelle kann daher als belegt gelten, dass es sich um eine absichtsvolle Angleichung der augusteischen Dichter an jene Seherinnen handelt, deren Bücher seit augusteischer Zeit im Inneren des palatinischen Tempels aufbewahrt wurden. Im weiteren Verlauf dieses Beitrages soll nun aber aufgezeigt werden, dass es sich bei dieser absichtsvollen Angleichung um weitaus mehr handelt als um einen schlichten Rückgriff auf einen in der griechischen Dichtung bereits zuvor angelegten Gedanken. Vielmehr existierten im unmittelbaren Lebensumfeld der augusteischen Dichter noch heute zu fassende Umstände, die sich im palatinischen Heiligtum manifestierten und die der besagten Angleichung eine besondere Qualität verliehen.

\section{Die Zeugnisse des Ovid und des Tacitus}

Es ist ein berühmtes Zeugnis des Ovid, das einen weiteren Einblick in das Phänomen gewährt. Im Jahr 8 n. Chr. wurde dieser jüngste der augusteischen Dichter aus Rom nach Tomis, einer kleinen Stadt an der Küste des Schwarzen Meeres, verbannt. Die genauen Hintergründe dieser Maßnahme werden in der Forschung noch immer viel diskutiert, sind für den gegebenen Kontext aber von untergeordnetem Interesse. Von wesentlich größerer Bedeutung ist die Tatsache, dass seine Verbannung Ovid keinesfalls davon abhielt, weitere Werke zu publizieren. Zu diesen gehört auch die Sammlung der sogenannten Tristia. Das erste Gedicht von deren drittem Buch wiederum enthält eine für unser Verständnis des palatinischen Heiligtums bedeutsame Passage:

Inde tenore pari gradibus sublimia celsis

ducor ad intonsi candida templa dei,

signa peregrinis ubi sunt alterna columnis,

Belides et stricto barbarus ense pater,

44 Hor. carm. 1,31,1f.; Nisbet \& Hubbard (1970: pp. 347-349); Della Casa (1995: p. 55); Miller (2009: pp. 52 und 225).

45 Prop. 2,10,19 und 4,6,1 und 4,6,10; Miller (2009: pp. 228-230).

46 Die Verwendung des Wortes vates anstelle von poeta kommt bei Ovid derart häufig vor, dass eine vollständige Aufzählung der Stellen den zweckmäßigen Rahmen sprengen würde. Siehe bspw.: Ov. am. 1,1,6 und $3,1,34$; Ov. met. 11,733 und 15,155 und 15,282 und 15,622 und 15,867 und 15,879 . Newman (1967: pp. 100-114).

47 Rüpke (2005: p. 1353); Keskiaho (2013: p. 150); Schnegg-Köhler (2020: p. 227).

48 Tib. 2,5,18 und 65 und 114. Ross (1975: p. 154); Della Casa (1995: pp. 55-56); Miller (2009: p. 245). 
quaeque uiri docto ueteres cepere nouique

pectore, lecturis inspicienda patent.

Quaerebam fratres, exceptis scilicet illis,

quos suus optaret non genuisse pater.

Quaerentem frustra custos me sedibus illis

praepositus sancto iussit abire loco.

„In dem gleichen ununterbrochenen Lauf werde ich dann die hochragenden Stufen zu dem erhabenen, strahlend weißen Tempel des ungeschorenen Gottes emporgeführt, wo sich Statuen mit fremdländischen Säulen abwechseln, Danaiden und ihr Barbarenvater mit gezücktem Schwert. Und alles, was Männer alter und neuer Zeit mit gelehrtem Verstand erdachten, steht dort dem Lesewilligen offen. Ich suchte meine Brüder, ausgenommen selbstverständlich jene, von denen ihr Vater wünschte, er hätte sie nie hervorgebracht. Mir vergeblich Suchendem befahl der dieser Stätte vorstehende Wächter, mich von dem heiligen Ort zu entfernen.“

Ov. trist. 3,1,59-68

Was Ovid hier beschreibt, ist die von ihm imaginierte Ankunft seines Buches aus Tomis in Rom. Dessen erstes Ziel ist die eingangs erwähnte berühmte augusteische Bibliothek im Heiligtum des Apollon. ${ }^{49}$ Dort aber erhält es keinen Einlass, sondern wird von dem zuständigen Wächter (custos), also dem Bibliothekar, zurückgewiesen. ${ }^{50}$ Diesem Zeugnis nach bestand ein Teil der Strafe Ovids darin, dass seine Bücher nicht mehr in der palatinischen Bibliothek aufbewahrt wurden. Demnach aber haben wir uns diese Bibliothek keinesfalls als eine Institution zu denken, welche so viel wie möglich von der zeitgenössischen Literatur ansammelte. Der Bibliothekar besaß vielmehr die Aufgabe - wobei er bis zu einem gewissen Grad sicher der persönlichen Weisung des Augustus folgte -, die tagesaktuelle Produktion aktiv zu beurteilen und nur solche Werke aufzunehmen, welche einem gewissen Anspruch genügten. ${ }^{51}$ Hieraus aber folgt, dass es sich für einen Dichter um eine große Ehre gehandelt haben muss, wenn seine Werke an diesem Ort akzeptiert wurden.

An dieser Stelle mag es nun zuerst schwerfallen, die Verbindung der Passage des Ovid zu den oben vorgestellten sibyllinischen Orakeln bzw. zu der Tatsache, dass sich die augusteischen Dichter als vates bezeichneten, zu erkennen. Durch die Einbeziehung eines weiteren Zeugnisses treten die Zusammenhänge allerdings deutlich zutage. Es handelt

49 Aus anderen Quellen erfahren wir, dass der Senat des Öfteren in diesem Gebäude tagte. Daher ist davon auszugehen, dass sich die Bibliothek auch im Inneren des templum des Heiligtums befand, da der Senat nur an einem solchen Ort tagen durfte. Aus diesem Grund beschreibt Ovid die Bibliothek vollkommen korrekt als locus sanctus; vgl.: Suet. Aug. 29; Suet. Dom. 20. Talbert (1984: pp. 113-114); Balensiefen (2002: pp. 102-105); Balensiefen (2004: pp. 103-111); Iacopi \& Tedone (2005/6: pp. 351-378); Miller (2009: p. 189); Tucci (2013: pp. 286-291); Fleury \& Madeleine (2019: pp. 171-179).

50 Während der augusteischen Epoche war sicher Gaius Iulius Hyginus mit dieser Aufgabe betraut. Wie genau hingegen eine Bemerkung des Sueton aufzufassen ist, die auch Pompeius Macer eine „Aufsicht über die öffentlichen Bibliotheken“ (cui ordinandas bibliothecas delegaverat) zuschreibt, ist Gegenstand einer Forschungsdebatte; Ov. trist. 3,14; Suet. gramm. 20; Suet. Iul. 56; vgl. auch Hor. Epist. 1,3,17. Christes (1979: pp. 74-76); Dix (1988: p. 28); White (1992: pp. 213-214); Dix (1994: pp. 289-290); Houston (2002: pp. 162 und 168); Houston (2008: p. 250).

51 Dix (1988: pp. 28-32); Dix (1994: pp. 289-290); Miller (2002: p. 138). 
sich um eine Passage aus den Annales des Tacitus. Der Historiker beschreibt dabei zwar einen Vorfall, welcher sich erst in der Zeit des Tiberius ereignete, der in vergleichbarer Form allerdings auch schon in den vorigen Jahrzehnten und Jahrhunderten des Öfteren vorgekommen sein muss:

Relatum inde ad patres a Quintiliano tribuno plebei de libro Sibullae, quem Caninius Gallus quindecimvirum recipi inter ceteros eiusdem vatis et ea de re senatus consultum postulaverat. quo per discessionem facto misit litteras Caesar, modice tribunum increpans ignarum antiqui moris ob iuventam. Gallo exprobrabat quod scientiae caerimoniarumque vetus incerto auctore ante sententiam collegii, non, ut adsolet, lecto per magistros aestimatoque carmine, apud infrequentem senatum egisset.

„Daraufhin wurde der Senat durch den Volkstribun Quintilianus über ein Buch der Sibylle unterrichtet, für das Caninius Gallus, einer der Quindecimviri, nach der Aufnahme unter die übrigen Bücher derselben Seherin und einem entsprechenden Senatsbeschluss über die Angelegenheit verlangt hatte. Als dieser durch einfache Abstimmung erfolgt war, schickte der Kaiser einen Brief, in dem er den Tribun mäßig tadelte, da er die alte Sitte wegen seiner Jugend nicht kenne. Dem Gallus aber warf er vor, er habe das Orakel, obwohl er ein Routinier in dem einschlägigen Fachgebiet und den heiligen Bräuchen sei, trotz seiner nicht sicher bestätigten Autorenschaft, ohne vorher ein Gutachten des Kollegiums einzuholen und es, wie üblich, durch dessen magistri lesen und prüfen zu lassen, vor den nur in geringer Zahl besetzten Senat gebracht.“

Tac. ann. 6, 12

Diese Passage stellt eine der wichtigsten Quellen für unser Verständnis des Wirkens und der Funktion der eng mit dem Gott Apollon verbundenen Priesterschaft der decem- bzw. quindecimviri dar. Wie oben bereits aufgezeigt, hatte Augustus im Jahr 12 v. Chr. Tausende zuvor frei in der Bevölkerung zirkulierende Orakelschriften verbrennen lassen. In den folgenden Jahren hatte sich diese Form der Literatur aber bereits wieder erholt. Ein solches Buch, dessen Verfasserschaft einer Sibylle zugeschrieben wurde, fiel in die Hände des Gallus, eines Mitgliedes der quindecimviri. Dieser versuchte daraufhin, seine Aufnahme in die offizielle Sammlung zu forcieren. Was Tacitus nicht betont, was wir aber aus den weiteren Quellen rekonstruieren können, ist, dass sich diese Sammlung aufgrund der oben erwähnten Maßnahmen des Augustus zu dieser Zeit bereits seit einigen Jahrzehnten im palatinischen Apollontempel befand. Gallus versuchte demnach das neu aufgefundene Buch im palatinischen Heiligtum unterzubringen. Hierfür tadelte ihn Tiberius. Doch bezog er sich nicht auf das eigentliche Vorhaben als solches. Denn im Wesentlichen hatte Gallus nichts Falsches getan. Sein Fehler bestand alleine darin, dass er zu vorschnell gehandelt hatte. Das Buch hätte nämlich zuerst, wie dies gewöhnlich im Rahmen derartiger Vorkommnisse zu geschehen pflegte (ut adsolet), von den magistri der quindecimviri gelesen und beurteilt werden müssen (lecto per magistros aestimatoque). ${ }^{52}$

Die großen Parallelen zwischen der Rolle der magistri des stark mit Apollon verbundenen Priesterkollegiums und jener des custos der Bibliothek des palatinischen Apol-

52 Vgl. Fischer (2020b: pp. 548-553). 
lonheiligtums sind deutlich zu erkennen: Sowohl der Bibliothekar als auch die Priester waren dafür verantwortlich, neue Bücher zu kontrollieren und zu beurteilen, bevor sie im Inneren des augusteischen Heiligtums aufgenommen wurden. Der hauptsächliche Unterschied zwischen den beiden Büchersammlungen tritt ebenso klar zutage. Eine von ihnen, jene der sibyllinischen Bücher, befand sich unmittelbar im Inneren des Tempels selbst. Diese Bücher beinhalteten dem zeitgenössischen Verständnis zufolge Orakel, welche ursprünglich auf von Apollon inspirierte Prophetinnen (vates) zurückgingen. Bei der anderen Sammlung, welche nur wenige Meter von dem Tempel entfernt aufbewahrt wurde, handelte es sich hingegen im Wesentlichen um eine herkömmliche Bibliothek. Diese stand allerdings mitten im literarischen und kulturellen Zentrum des augusteischen Roms. Die Tatsache, dass die augusteischen Dichter, für welche es eine große Ehre gewesen sein muss, wenn ihre Werke in diese Bibliothek aufgenommen wurden, während eben dieser Epoche damit begannen, sich selbst als vates zu bezeichnen, muss vor diesem Hintergrund beurteilt werden.

\section{Fazit}

Vor ein abschließendes Fazit sei eine Zusammenfassung der wesentlichen Punkte der vorgelegten Untersuchung gestellt. Das palatinische Apollonheiligtum befand sich sowohl in geographischer als auch in kultureller Hinsicht im Zentrum des augusteischen Roms. Sein Tempel sowie die nur wenige Meter von ihm entfernte Bibliothek bilden ein häufig wiederkehrendes Motiv der zeitgenössischen Autoren. Ein Zeugnis des Ovid belegt, dass es für die Werke dieser Männer keineswegs eine Selbstverständlichkeit darstellte, im Inneren dieser Bibliothek aufgenommen zu werden. Vielmehr war es die Aufgabe des Bibliothekars, eine Auswahl zu treffen. Das Innere des Tempels wiederum beherbergte seit der augusteischen Epoche die sibyllinischen Bücher. Bei diesen handelte es sich um eine Form hexametrischer Dichtung, von der mitunter auch Werke frei in der Bevölkerung zirkulierten. Mit dieser Sammlung beauftragt zeigte sich das Priesterkollegium der quindecimviri sacris faciundis, welches stark mit dem Gott Apollon assoziiert wurde. Dabei war es dem Zeugnis des Tacitus zufolge ebenso die Aufgabe der magistri dieses Kollegiums, neu aufgefundene Orakelschriften zu prüfen, bevor sie in die Sammlung im palatinischen Tempel integriert wurden, wie es die Aufgabe des Bibliothekars der palatinischen Bibliothek war, die zeitgenössische Literaturproduktion zu beurteilen, bevor einzelne Werke ihren Weg in die Bibliothek fanden. Zudem war es exakt diese Zeit, in welcher sich dieser überaus enge Zusammenhang zwischen Bibliothek, Tempel und Priesterkollegium etablierte, als die zeitgenössischen Dichter damit begannen, sich selbst als vates zu bezeichnen.

Sowohl das palatinische Apollonheiligtum selbst als auch die Performanz der Priesterschaft etwa im Rahmen wiederkehrender Feste wie der ludi Apollinares prägten das Stadtbild des augusteischen Roms. Bei den dargelegten Zusammenhängen handelt es sich demnach um Gegebenheiten, welche den Zeitgenossen tagtäglich vor Augen standen und somit einen festen Bestandteil des kontemporären Diskurses bildeten. Bekanntlich 
sind es gerade derartige, für die unmittelbaren Zeitgenossen überaus selbstverständliche Zusammenhänge, die von unseren Quellen nicht extra hervorgehoben werden und von der modernen Forschung daher mühselig rekonstruiert werden müssen. Doch einzig und alleine ihre bessere Kenntnis schafft ein tieferes Verständnis der unmittelbaren Kontexte, unter deren Einfluss unsere Quellen ursprünglich entstanden, und bildet somit ein solides Fundament für weiterführende Interpretationen solch komplexer Phänomene wie der Selbstbenennung der augusteischen Dichter als vates. Wie im Rahmen dieses Beitrages aufgezeigt wurde, ist es hierfür allerdings unabdingbar notwendig, historische, archäologische und philologische Quellen und Forschungsergebnisse miteinander zu kombinieren und im gleichem Maße zu berücksichtigen.

\section{Bibliographie}

Babcock, C. L. (1967). Horace Carm. 1. 32 and the Dedication of the Temple of Apollo Palatinus. Classical Philology, 62, 189-194.

Balensiefen, L. (2002). Die Macht der Literatur. Über die Büchersammlungen des Augustus auf dem Palatin. In W. Hoepfner (Ed.), Antike Bibliotheken (pp. 97-116). Mainz: Philipp von Zabern.

Balensiefen, L. (2004). Bibliotheca Palatina - Die Apollo-Bibliothek. In A. Hoffmann, \& U. Wulf (Eds.), Die Kaiserpaläste auf dem Palatin in Rom. Das Zentrum der römischen Welt und seine Bauten (pp. 100-111). Mainz: Philipp von Zabern.

Balensiefen, L. (2009). Apollo Palatinus. Ein Kultgründungsvorhaben des jungen Caesar Divi Filius. In C. Schmitz, \& A. Bettenworth (Eds.), Mensch - Heros - Gott. Weltentwürfe und Lebensmodelle im Mythos der Vormoderne (pp. 67-89). Stuttgart: Franz Steiner.

Beard, M., North, J., \& Price, S. (1998). Religions of Rome, I.: A History. Cambridge: Cambridge University Press.

Bickel, E. (1951). Vates bei Varro und Vergil. Die Kult- und Ahnenlieder, Seher-, Zauber- und Heilverse des vates. Rheinisches Museum für Philologie, 94, 257-314.

Bremmer, J. (1993). Prophets, Seers, and Politics in Greece, Israel, and Early Modern Europe. Numen, 40, 150-183.

Christes, J. (1979). Sklaven und Freigelassene als Grammatiker und Philologen im antiken Rom. Wiesbaden: Franz Steiner.

Dahlmann, H. (1948). Vates. Philologus, 97, 337-353.

Davis, J. P. (2001). The Fabrication of Tradition: Horace, Augustus and the Saecular Games. Ramus, 30, 111-127.

Della Casa, A. (1995). L'uso del termine poeta a Roma nell'eta augustea. In L. Belloni, G. Milanese, \& A. Porro (Eds.), Studia classica Iohanni Tarditi oblata (pp. 51-62). Milan: Vita e Pensiero.

Dix, T. K. (1988). Ovid strikes out: Tristia 3.1 and the first public libraries at Rome. The Augustan Age, 8, 27-35.

Dix, T. K. (1994). "Public Libraries" in Ancient Rome: Ideology and Reality. Libraries E Culture, 29, 282-296.

Dräger, O. (1994). Religionem significare. Studien zu reich verzierten römischen Altären und Basen aus Marmor. Mainz: Philipp von Zabern. 
Février, C. (2002). De l'usage des Livres: le décemvir, prètre ou vates? Latomus, 61, 821-841.

Fischer, J. (2020a). Augustus und Apollon. Notizen zu den Hintergründen einer ,göttlichen‘ Beziehung. In J. Bartz, M. Müller, \& R. F. Sporleder (Eds.), Augustus immortalis. Aktuelle Forschungen zum Princeps im interdisziplinären Diskurs. Beiträge des interdisziplinären Symposions an der Humboldt-Universität zu Berlin, 25.-27. Oktober 2019 (pp. 131-138). Berlin: Humboldt-Universität.

Fischer, J. (2020b). Q. Fabius Pictor, das Orakel von Delphi und die sibyllinischen Bücher Roms. Zur Rolle von Orakeln in Rom und Griechenland. Gymnasium, 127, 535-567.

Fischer, J. (forthcoming). Folia ventis turbata. Sibyllinische Orakel und der Gott Apollon zwischen später Republik und augusteischem Principat (Studien zur Alten Geschichte).

Fleury, P., \& Madeleine, S. (2019). Le sanctuaire d'Apollon, trait d'union entre le Palatin d'Auguste et celui des Flaviens. Proposition de restitution virtuelle. In M. de Souza, \& O. Devillers (Eds.), Neronia X. Le Palatin. Émergence de la Colline du Pouvoir à Rome (Ausonius Èditions Mémoires, 55; pp. 167-182). Bordeaux: Ausonius.

Gagé, J. (1931). Les sacerdoces d'Auguste et ses réformes religieuses. Mélanges d'Archéologie et d'Histoire de l'École française de Rome, 48, 75-108.

Gagé, J. (1955). Apollon romain. Essai sur le culte d'Apollon et le développement du 'ritus Graecus' a Rome des origines à Auguste (Bibliothèque des Écoles Françaises d'Athènes et de Rome, 182). Paris: E. de Boccard.

Gauger, J. D. (2002). Sibyllinische Weissagungen. Düsseldorf - Zürich: Artemis \& Winkler.

Gillmeister, A. (2015). Cultural Paraphrase in Roman Religion in the Age of Augustus. The Case of the Sibyl and the Sibylline Books. Acta Antiqua Academiae Scientiarum Hungaricae, 55, 211-222.

Gillmeister, A. (2019). The Guardians of the Sibylline Books. The Viri Sacris Faciundis College in Roman Religion. Lugano: Agorà \& Co.

Götte, H. R. (1984). Corona Spicea, Corona Civica und Adler. Bemerkungen zu drei römischen Dreifussbasen. Archäologischer Anzeiger, 1984, 573-589.

Graf, F. (1985). Nordionische Kulte. Religionsgeschichtliche und epigraphische Untersuchungen zu den Kulten von Chios, Erythrai, Klazomenai und Phokaia (Bibliotheca Helvetica Romana, 21). Rom: Schweizerisches Institut.

Graf, F. (2009). Apollo. London - New York: Routledge.

Guittard, Ch. (2007). Carmen et prophéties a Rome (Recherches sur les Rhétoriques Religieuses, 6). Turnhout: Brepols.

Hall, J. M. (2014). Artifact and Artifice. Chicago - London: The University of Chicago Press.

Hekster, O., \& Rich, J. (2006). Octavian and the Thunderbolt: the Temple of Apollo Palatinus and Roman Tradition of Temple Building. The Classical Quarterly, 56, 149-168.

Houston, G. W. (2002). The Slave and Freedman Personnel of Public Libraries in Ancient Rome. Transactions of the American Philological Association, 132, 139-176.

Houston, G. W. (2008). Tiberius and the Libraries: Public Book Collections and Library Buildings in the Early Roman Empire. Libraries E the Cultural Record, 43, 247-269.

Humphrey, J. (1986). Roman circuses: arenas for chariot racing. Berkeley - Los Angeles: University of California Press.

Iacopi, I., \& Tedone, G. (2005/6). Biblioteca e Porticus ad Apollinis. Römische Mitteilungen, 112, 351-378.

Jocelyn, H. D. (1995). 'Poeta' and 'Vates': Concerning the Nomenclature of the Composer of 
Verses in Republican and Early Imperial Rome. In L. Belloni, G. Milanese, \& A. Porro (Eds.), Studia classica Iohanni Tarditi oblata (pp. 19-50). Milan: Vita e Pensiero.

Kellum, B. (1985). Sculptural Programs and Propaganda in Augustan Rome. The Temple of Apollon on the Palatine. Archaeologica Transatlantica, 5, 169-176.

Keskiaho, J. (2013). Re-visiting the Libri Sibyllini. Some Remarks on Their Nature in Roman Legend and Experience. In M. Kajava (Ed.), Studies in Ancient Oracles and Divination (Acta Instituti Romani Finlandiae, 40; pp. 145-172). Rome: Institutum Romanum Finlandiae.

Künzl, E. (1988). Der römische Triumph. Siegesfeiern im antiken Rom. München: C. H. Beck.

Le Doze, P. (2016). Vox Apollinis / Vox Augusti: liberté d'inspiration des poètes et principat augustéen. In S. Luciani, \& P. Zuntow (Eds.), Entre mots et marbre. Les métamorphoses d'Auguste (pp. 85-104). Bordeaux: Ausonius.

Lefèvre, E. (1989). Das Bildprogramm des Apollontempels auf dem Palatin (Xenia, 24). Konstanz: Universitätsverlag.

Lightfoot, J. L. (2007). The Sibylline Oracles. With Introduction, Translation, and Commentary on the First and Second Books. Oxford: Oxford University Press.

Martini, C. (1988). Base Triangolare (inv. n. 669). In A. Giuliano (Ed.), Museo Nazionale Romano. Le Sculture (Bd. I,2; pp. 182-184). Rom: De Luca.

Miller, J. F. (2002). Ovid on the Augustan Palatine (Tristia 3, 1). In J. F. Miller, C. Damon, \& K. Sara Myers (Eds.), Vertis in usum. Studies in honor of Edward Courtney (Beiträge zur Altertumskunde, 161; pp. 129-139). München - Leipzig: K. G. Saur.

Miller, J. F. (2004/5). Ovid and Augustan Apollo. Hermathena, 177/178, 165-180.

Miller, J. F. (2009). Apollo, Augustus, and the Poets. Cambridge: Cambridge University Press.

Mlasowsky, A. (2010). Ara Pacis. Ein Staatsmonument des Augustus auf dem Marsfeld. Mainz: Philipp von Zabern.

Monaca, M. (2005). La Sibilla a Roma, I libri sibillini fra religione e politica. Consenza: Edizioni Lionello Giordano.

Nagy, G. (1990). Ancient Greek Poetry, Prophecy, and Concepts of Theory. In J. L. Kugel (Ed.), Poetry and Prophecy. The Beginnings of a Literary Tradition (pp. 56-64). Ithaca - London: Cornell University Press.

Newman, J. K. (1967). The Concept of Vates in Augustan Poetry (Collection Latomus, 89). Brussels: Latomus.

Nicholls, M. (2018). Libraries and Literary Culture in Rome. In A. Claridge, \& C. Holleran (Eds.), A Companion to the City of Rome (pp. 343-361). Malden MA: John Wiley \& Sons.

Nisbet, R. G. M., \& Hubbard, M. (1970). A Commentary on Horace. Book 1. Oxford: Oxford University Press.

Parke, H. W. (1988). Sibyls and Sibylline Prophecy in Classical Antiquity (Croom Helm Classical Studies). London: Routledge.

Potter, D. S. (1990). Sibyls in the Greek and Roman World (Review of the book Sibyls and Sibylline Prophecy in Classical Antiquity, by H. W. Parke). Journal of Roman Archaeology, 3, 471-483.

Radke, G. (1963). Quindecimviri. Realencyclopädie der Classischen Altertumswissenschaft, 24, 1114-1148. Rendini, P. (1988). Base Triangolare a Bordi Concavi (inv. n. 212207). In A. Giuliano (Ed.), Museo Nazionale Romano. Le Sculture (Bd. I,2; pp. 233-236). Rom: De Luca.

Roessli, J.-M. (2004). Catalogues de sibylles, recueil(s) de Libri Sibyllini et corpus des Oracula 
Sibyllina. In E. Norelli (Ed.), Recueils normatifs et canons dans l' Antiquité (pp. 47-68). Lausanne: Éditions du Zèbre.

Ross, D. O. (1975). Backgrounds to Augustan Poetry: Gallus Elegy and Rome. Cambridge: Cambridge University Press.

Rüpke, J. (2005). Fasti sacerdotum. Die Mitglieder der Priesterschaften und das sakrale Funktionspersonal römischer, griechischer, orientalischer und jüdisch-christlicher Kulte in der Stadt Rom von 300 v. Chr. bis 499 n. Chr. (Teil 1-3). Stuttgart: Franz Steiner.

Rüpke, J. (2006). Die Religion der Römer. München: Beck.

Ryan, F. X. (2006). Die Apollinarspiele zur Zeit der Republik. Aevum, 80, 67-104.

Ryan, F. X. (2006/7). Die Legende IMP.AV.X auf den Denaren des Triumvirn Caldus. Schweizer Münzblätter, 56-57, 39-42.

Santi, C. (2008). Sacra facere. Aspetti della prassi ritualistica divinatoria nel mondo romano. Rom: Bulzoni Editore.

Scheid, J. (2009). To Honour the Princeps and Venerate the Gods: Public Cult, Neighbourhood Cults, and Imperial Cult in Augustan Rome. In J. Edmondson (Ed.), Augustus (pp. 275-299). Edinburgh: Edinburgh University Press.

Schnegg-Köhler, B. (2020). Die Inschriften zu den Ludi Saeculares. Acta ludorum saecularium. Berlin - Boston: De Gruyter.

Schröder, S. (1990). Plutarchs Schrift De Pythiae oraculis. Text, Einleitung und Kommentar (Beiträge zur Altertumskunde, 8). Stuttgart: Teubner.

Sfameni Gasparro, G. (1998). La Sibylla Voce del Dio per Pagani, Ebrei e Christiani: Un Modulo Profetico al Crocevia delle Fedi. In I. Chirassi Colombo, \& A. Seppilli (Eds.), Sibille e Linguaggi oracolari (Istituti editoriali e poligrafici internazionali, 3; pp. 505-553). Pisa - Rom: Ist Editoriali E Poligrafici Libri.

Sfameni Gasparro, G. (2002). Oraculi, profeti, sibille. Rivelazione e salvezza nel mondo antico (Biblioteca di Scienze Religiose, 171). Rom: Libreria Ateneo Salesiano.

Siebert, A. V. (1999). Instrumenta Sacra. Untersuchungen zu römischen Opfer-, Kult- und Priestergeräten (Religionsgeschichtliche Versuche und Vorarbeiten, 44). Berlin - New York: De Gruyter.

Simon, E. (1986). Augustus. Kunst und Leben in Rom um die Zeitenwende. München: Hirmer.

Simon, E. (2012). Ara Pacis Augustae. Der Altar der Friedensgöttin Pax Augusta in Rom (Schriften des Knauf-Museums Iphofen, 3). Dettelbach: J. H. Kröll.

Suarez de la Torre, E. (1994). Sibylles, mantique inspireé et collections oraculaires. Kernos, 7, 179-205.

Talbert, R. J. A. (1984). The Senate of Imperial Rome. Princeton - New Jersey: Princeton University Press.

Tucci, P. L. (2013). Flavian libraries in the city of Rome. In J. König, K. Oikonomopoulou, \& G. Woolf (Eds.), Ancient Libraries (pp. 277-312). Cambridge: Cambridge University Press.

Wardle, D. (2014). Suetonius. Life of Augustus. Oxford: Oxford University Press.

White, P. (1992). 'Pompeius Macer' and Ovid. Classical Quarterly, 42, 210-218.

White, P. (1993). Promised Verse. Poets in the Society of Augustan Rome. Cambridge MA: Harvard University Press.

Zanker, P. (1983). Der Apollontempel auf dem Palatin. Ausstattung und politische Sinnbezüge nach der Schlacht von Actium. In K. de Fine Licht (Ed.), Città e architettura nella Roma imperiale: 
Atti del seminario del 27 ottobre 1981 nel 25anniversario dell'Accademia di Danimarca (Analecta Romana, Supplementum, 10; pp. 21-40). Odense: Odense University Press.

Zanker, P. (2009). Augustus und die Macht der Bilder. München: C. H. Beck.

Zwierlein-Diehl, E. (2012). Antike Gemmen und ihr Nachleben. Berlin - New York: De Gruyter.

Dr. Jens Fischer / fischerj@uni-potsdam.de

Institute of Ancient Studies, Department of Ancient History

Martin-Luther-University Halle-Wittenberg, Faculty of Philosophy I

Universitätsplatz 12, 06108 Halle/Saale, Germany

This work can be used in accordance with the Creative Commons BY-SA 4.0 International license terms and conditions (https://creativecommons.org/licenses/by-sa/4.0/legalcode). This does not apply to works or elements (such as image or photographs) that are used in the work under a contractual license or exception or limitation to relevant rights 